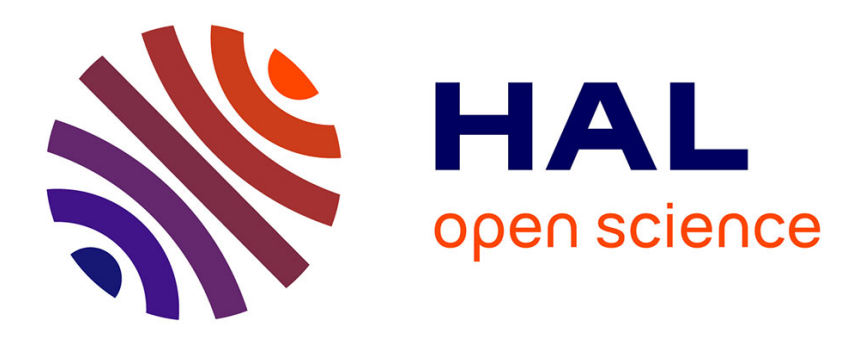

\title{
Ordered categories and ordered semigroups
}

Jean-Eric Pin, Arnaud Pinguet, Pascal Weil

\section{To cite this version:}

Jean-Eric Pin, Arnaud Pinguet, Pascal Weil. Ordered categories and ordered semigroups. Communications in Algebra, 2002, 30, pp.5651-5675. hal-00112616

\section{HAL Id: hal-00112616 https://hal.science/hal-00112616}

Submitted on 9 Nov 2006

HAL is a multi-disciplinary open access archive for the deposit and dissemination of scientific research documents, whether they are published or not. The documents may come from teaching and research institutions in France or abroad, or from public or private research centers.
L'archive ouverte pluridisciplinaire HAL, est destinée au dépôt et à la diffusion de documents scientifiques de niveau recherche, publiés ou non, émanant des établissements d'enseignement et de recherche français ou étrangers, des laboratoires publics ou privés. 


\title{
Ordered categories and ordered semigroups
}

\author{
Jean-Eric Pin*, Arnaud Pinguet, and Pascal Weil ${ }^{\dagger}$ \\ Jean-Eric.Pin@liafa.jussieu.fr, apinguet@clipper.ens.fr, \\ Pascal. Weil@labri.u-bordeaux.fr ${ }^{\ddagger}$
}

\begin{abstract}
We use ordered categories to study semidirect decompositions of finite ordered semigroups. We obtain ordered analogues of the derived category theorem and of the delay theorem. Next we prove that the ordered analogues of semilattices and of $\mathcal{J}$-trivial monoids constitute local varieties, and we derive some decomposition theorems from these results.
\end{abstract}

In [10], Pin and Weil initiated a study of the semidirect decomposition of finite ordered semigroups. We refer the reader to the introduction of that article for details on the motivations for such a study, especially in connection with formal language theory. In another paper [11], Pin and Weil give some applications of semidirect decomposition results to language theory, some of which depend on results from the present article.

The foundations for the study of the semidirect product of ordered semigroups, are given in [10], as are some interesting decomposition results for classes of finite ordered semigroups, notably regarding naturally ordered inverse monoids, ordered monoids in which the unit is the maximum element, and ordered monoids in which the unit is maximum among the idempotents.

In order to go further towards decomposing varieties of ordered semigroups and monoids, we need to extend our scope and to consider finite ordered semigroupoids and categories, just like in the decomposition theory of unordered semigroups and monoids. Semigroupoids are defined like

\footnotetext{
${ }^{*}$ LIAFA, Université Paris VII and CNRS, Case 7014, 2 Place Jussieu, 75251 Paris Cedex 05, France

${ }^{\dagger}$ LaBRI, Université Bordeaux I and CNRS, 351 cours de la Liberation, 33405 Talence Cedex, France

${ }^{\ddagger}$ Work supported by INTAS project 1224 .
} 
categories, only dropping the requirement that there exist a unit at each object. In the unordered case, this approach was introduced by Tilson [20], following pioneering work of Tilson [19], Nico [7], Thérien and Weiss [17] and Margolis and Pin [6]. It gave rise to numerous applications, and to foundational papers such as Almeida and Weil's [1], Jones and Pustejovsky's [3], Rey's [12, 13, 14], Teixeira's [16], etc.

There is no particular difficulty in defining varieties of ordered semigroupoids and categories, and we prove in Section 1 that the varieties of ordered categories can be defined by strongly connected graph identities, an ordered version of Tilson's so-called Bonded Component Theorem [20].

Section 2 is devoted to the ordered version of the derived semigroupoid theorem, whose unordered version is again due to Tilson [20]. This theorem characterizes the situations where a relational morphism $\varphi$ from an ordered semigroup into an unordered semigroup $T$ can be factorized through a semidirect product $V * T$ or a wreath product $V \circ T$ ( $V$ ordered), in terms of an ordered semigroupoid associated with $\varphi$, called the derived semigroupoid of $\varphi$.

Just like in the unordered case, the ordered derived semigroupoid theorem emphasizes the importance of the notion of local varieties $\mathbf{V}$ of monoids. A variety $\mathbf{V}$ of ordered semigroups is said to be local if, whenever the loop semigroups of a finite semigroupoid $C$ are in $\mathbf{V}$ (we say that $C \in \ell \mathbf{V}$ ), then $C$ divides a semigroup in $\mathbf{V}(C \in \mathbf{g V})$. The decidability of the membership problem for varieties of the form $\mathbf{V} * \mathbf{W}$ (with $\mathbf{W}$ unordered) depends in part on the membership problem for $\mathbf{g V}$. In general, this is a difficult problem; however, if $\mathbf{V}$ is decidable and local, then $\mathbf{g} \mathbf{V}$ is simply seen to be decidable.

In Section 3, we prove the ordered delay theorem, which is a case in point: it reduces the membership problem of $\mathbf{V} * \mathbf{L I}$, where $\mathbf{V}$ is a variety of ordered monoids and $\mathbf{L I}$ is the variety of locally trivial semigroups, to the membership problem in $\mathbf{g V}$. The decidability of $\mathbf{V} * \mathbf{L I}$ is proved for a large class of varieties $\mathbf{V}$ : in fact, as soon as $\mathbf{V}$ is decidable and local, or $\mathbf{V}$ is decidable and contains a certain 6-element ordered monoid.

The last section is a study of the locality of two simple, yet important varieties of ordered monoids: the class $\mathbf{J}^{+}$of ordered monoids in which 1 is maximum, and the class $\mathbf{J}_{1}^{+}$of idempotent and commutative elements of $\mathbf{J}^{+}$. These classes are ordered analogues of the class $\mathbf{J}$ of $\mathcal{J}$-trivial monoids and of the class $\mathbf{J}_{1}$ of semilattices (see [9] for details), which play an important role in formal language theory [8]. These classes also occurred as factors in semidirect decompositions in [10]. Not surprisingly, $\mathbf{J}_{1}^{+}$is a local variety, just like its unordered counterpart $\mathbf{J}_{1}$ was one of the first varieties to be proved to be local. It is more surprising that $\mathbf{J}^{+}$is local as well, as $\mathbf{J}$ is the 
typical example of a non-local variety [5, 18].

Both these locality results can be used, in conjunction with the delay theorem, to give semidirect decompositions of the class of finite ordered semigroups that are locally in $\mathbf{J}_{1}^{+}$and in $\mathbf{J}^{+}$respectively. The latter, $\mathbf{L} \mathbf{J}^{+}$, which is also denoted $\mathbf{B}_{1}^{+}$, plays a crucial role in the study of the concatenation product of languages and of the dot-depth hierarchy, see $[4,5,9]$.

Before we embark into the paper, let us emphasize again that we deal here only with semidirect decompositions of ordered semigroups and monoids using products of the form $V * T$ where $V$ is ordered and $T$ is not. The main tool we introduce, namely the ordered derived semigroupoid of a relational morphism $\varphi: S \rightarrow T$, depends on the order on $S$ but not on that on $T$. Some of the semidirect decomposition results in [11] have both semidirect factors ordered, but these results do not use the ordered derived semigroupoid. It may be the case that future applications will require the development of category-theoretic tools taking into account the order relations on both the domain and the target of a relational morphism; however, the tools described here suffice for the language-theoretic applications which we have in mind, and which are described in [11].

\section{Ordered semigroupoids and categories}

In this section we give the basic definitions regarding finite ordered semigroupoids and categories and their varieties, and we show that every such variety can be defined by a set of strongly connected identities.

\subsection{Elementary definitions}

Recall that a graph $C$ consists of the following data: a set of objects, or vertices, written $\operatorname{Obj}(C)$; and for every pair $(u, v)$ of objects of $C$, a set $C(u, v)$ of arrows from $u$ to $v$.

Let $x, y$ be arrows of $C$. We say that $x$ and $y$ are coterminal if there exist objects $u, v$ of $C$ such that $x, y \in C(u, v)$; they are consecutive arrows if $x \in C(u, v)$ and $y \in C(v, w)$ for some objects $u, v, w$; and $x$ is a loop around $u$ if $u$ is an object and $x \in C(u, u)$. 

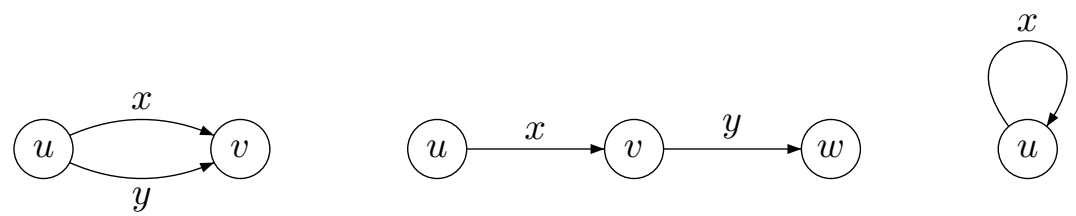

Figure 1: Coterminal arrows, consecutive arrows and a loop.

A composition rule on the graph $C$, assigns to each pair $(x, y)$ of consecutive arrows $x \in C(u, v)$ and $y \in C(v, w)$ an arrow $x y \in C(u, w)$. We say the composition rule is associative if whenever $t, u, v, w$ are objects of $C$ and $x \in C(t, u), y \in C(u, v)$ and $z \in C(v, w)$, then $x(y z)=(x y) z$. A graph equipped with an associative composition rule is called a semigroupoid. A local identity at object $u$ is an element $1_{u} \in C(u, u)$ such that, for every object $v, x 1_{u}=x$ for every $x \in C(v, u)$ and $1_{u} x=x$ for every $x \in C(u, v)$. If $C$ admits a local identity at every object, then we say that $C$ is a category.

Here we view semigroupoids and categories as generalizations of semigroups and monoids: it is immediately verified that a one-object semigroupoid (resp. category) is a semigroup (resp. a monoid). A semigroupoid (resp. category) $C$ is trivial if every set of arrows $C(u, v)$ has cardinality at most 1 .
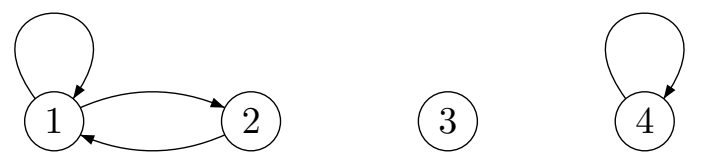

Figure 2: A trivial category

Let $X$ be a graph. The free semigroupoid on $X$ is denoted by $X^{+}$: it has the same object set as $X$, and its arrows are the non-empty paths in $X$, with obvious composition. Allowing also empty paths around each vertex yields the free category on $X$, written $X^{*}$.

We say that a relation $\leq$ on the set of arrows of a semigroupoid $C$, is compatible if the following properties are satisfied:

(1) if $x \leq y$, then $x$ and $y$ are coterminal;

(2) if $x, y$ are coterminal arrows and $x, z$ are consecutive arrows, and if $x \leq y$, then $x z \leq y z$

(3) if $x, y$ are coterminal arrows and $z, x$ are consecutive arrows, and if $x \leq y$, then $z x \leq z y$.

If the semigroupoid $C$ is equipped with a compatible order relation, we say that $C$ is an ordered semigroupoid. A compatible equivalence relation is called a congruence of semigroupoid. 


\subsection{Relational morphisms and varieties}

Let $C$ and $D$ be semigroupoids. Let us recall the following definitions, from $[20,1]$. A relational morphism $\tau: C \rightarrow D$ is given by a mapping $\tau: \operatorname{Obj}(C) \rightarrow \operatorname{Obj}(D)$ and for each pair $(u, v)$ of objects of $C$, by a relation $\tau: C(u, v) \rightarrow D(\tau(u), \tau(v))$ such that

(1) $\tau(x) \tau(y) \subseteq \tau(x y)$ for any consecutive arrows $x$ and $y$ of $C$,

(2) $\tau(x)$ is non-empty for any arrow $x$.

If $\tau(x)$ is a singleton for each arrow $x$, then $\tau$ is a morphism. It is said to be faithful if it is injective on each set $C(u, v)$, and to be a quotient if it is onto, and a bijection on the set of objects. We say that a relational morphism $\tau: C \rightarrow D$ is a division (of semigroupoids) if it satisfies the following injectivity condition:

(3) if $x$ and $y$ are coterminal arrows such that $\tau(x) \cap \tau(y) \neq \emptyset$, then $x=y$. It is known (see for instance [1]) that there exists a division from $C$ to $D$ if and only if there exists a semigroupoid $E$, a faithful morphism from $E$ to $D$, and a quotient morphism from $E$ to $C$.

If $(C, \leq)$ and $(D, \leq)$ are ordered semigroupoids and if $\tau: C \rightarrow D$ is a morphism, we say that it is a morphism of ordered categories if whenever $x$ and $y$ are coterminal arrows of $C$ and $x \leq y$, then $\tau(x) \leq \tau(y)$. We say that the morphism $\tau$ is ordered-faithful if, in addition, $\tau(x) \leq \tau(y)$ implies $x \leq y$.

If $\tau: C \rightarrow D$ is a relational morphism, we say that $\tau$ is a division of ordered semigroupoids if, whenever $x$ and $y$ are coterminal arrows and $x^{\prime} \leq y^{\prime}$ for some $x^{\prime} \in \tau(x)$ and $y^{\prime} \in \tau(y)$, then $x \leq y$.

It is easily verified that any division of ordered semigroupoids is also a division of semigroupoids. Also one verifies that there exists a division of ordered semigroupoids from $C$ to $D$ if and only if there exists an ordered semigroupoid $E$, an ordered-faithul morphism from $E$ to $D$ and a quotient morphism of ordered semigroupoids from $E$ to $C$.

This definition leads to that of a variety of ordered categories and that of a variety of ordered semigroupoids. A variety of ordered categories is a class of finite ordered categories containing the one-element semigroup, which is closed under taking finite direct products and under division (of ordered categories). As in the unordered case [20], these closure properties need to be slightly strengthened to obtain a satisfactory definition of a variety of semigroupoids: a variety of ordered semigroupoids is a class of finite ordered semigroupoids containing the one-element semigroup, which is closed under taking finite direct products and coproducts, and closed under division of or- 
dered semigroupoids (for categories, coproducts can be obtained as divisors of direct products).

If $\mathbf{V}$ is a variety of ordered semigroups (resp. monoids), we let $\mathbf{g V}$ be the variety of ordered semigroupoids (resp. categories) generated by $\mathbf{V}$, that is, the class of ordered semigroupoids (resp. categories) which divide an element of $\mathbf{V}$. We also let $\ell \mathbf{V}$ be the variety of all ordered semigroupoids (resp. categories) $C$ which are locally in $\mathbf{V}$, that is, for each object $u$ of $C$, the ordered semigroup (resp. monoid) $C(u, u)$ lies in $\mathbf{V}$. As in the unordered case, these varieties play a particular role.

It is immediate that $\ell \mathbf{V}$ is decidable if $\mathbf{V}$ is, and that $\mathbf{g V}$ is contained in $\ell \mathbf{V}$. The converse inclusion is not true in general. As we will see throughout this paper, the decidability of $\mathbf{g V}$ has important consequences, and it can be ascertained in a number of cases. We say that a variety $\mathbf{V}$ of ordered semigroups or monoids is local if $\mathbf{g} \mathbf{V}=\ell \mathbf{V}$. If $\mathbf{V}$ is decidable and local, then $\mathbf{g V}$ is clearly decidable. In the unordered case, it is well-known that the varieties of monoids $\mathbf{G}$ and $\mathbf{J}_{1}$ are local. The first result is elementary and can be found in [20]. The second one is due to Simon, (see [20, Section 15]). We will see other important examples of local varieties in Section 4. On the other hand, it is known that $\mathbf{J}$ is not a local variety. A famous result of Knast characterizes membership in $\mathbf{g} \mathbf{J}$ by a decidable condition on the 2-object subcategories [5, 18].

Like in the unordered case, the decidability of $\mathbf{g V}$ follows from that of $\mathbf{V}$ for a large class of varieties, see [20, Proposition 13.4] and [15]. Let $B_{2}^{+}$ be the 6 -element ordered monoid $B_{2}^{+}=\{0,1, a, b, a b, b a\}$ given by $a b a=a$, $b a b=b$ and $a^{2}=b^{2}=0$, and in which the order relation is given by $s \leq t$ if and only if $s=t$ or $s=0$. More generally, if $Q$ is a finite non-empty set, we let $B_{Q}^{+}$be the ordered monoid $B_{Q}^{+}=\{0,1\} \cup(Q \times Q)$ whose multiplication is given by

$$
(p, q)\left(p^{\prime}, q^{\prime}\right)= \begin{cases}\left(p, q^{\prime}\right) & \text { if } q=p^{\prime} \\ 0 & \text { otherwise }\end{cases}
$$

and whose order is given by $s \leq t$ if and only if $s=t$ or $s=0$. It is not difficult to verify that $B_{Q}^{+}$divides a direct product of copies of $B_{2}^{+}$.

We first prove a technical result. Let $C$ be a category. The consolidated semigroup $C_{c d}$ is the set

$$
C_{c d}=\{0\} \cup\{(u, s, v) \mid u, v \in \operatorname{Obj}(C), s \in C(u, v)\}
$$

equipped with the product

$$
(u, s, v)\left(u^{\prime}, s^{\prime}, v^{\prime}\right)= \begin{cases}\left(u, s s^{\prime}, v^{\prime}\right) & \text { if } v=u^{\prime} \\ 0 & \text { otherwise }\end{cases}
$$


If $C$ is an ordered category, $C_{c d}$ is ordered by letting $0 \leq s$ for each $s \in C_{c d}$, and $(u, s, v) \leq\left(u^{\prime}, s^{\prime}, v^{\prime}\right)$ if and only if $u=u^{\prime}, v=v^{\prime}$ and $s \leq s^{\prime}$. Next the monoid $C_{c d}^{1}=C_{c d} \cup\{1\}$ is ordered in such a way that the added unit 1 is not $\leq$-comparable to any other element (as in [10, Section 2.2]). It is immediate that $C$ divides $C_{c d}^{1}$.

Lemma 1.1 Let $C$ be an ordered category and let $M$ be an ordered monoid divided by $C$. Then $C_{c d}^{1}$ divides $M \times B_{Q}^{+}$.

Proof. The proof is the same as the analogous result in $[15,20]$. Let $\varphi: C \rightarrow$ $M$ be a division of ordered categories. We define $\varphi^{\prime}: C_{c d}^{1} \rightarrow M \times B_{Q}^{+}$by letting

$$
\begin{aligned}
\varphi^{\prime}(u, s, v) & =\varphi(s) \times\{(u, v)\} \\
\varphi^{\prime}(1) & =\{(1,1)\} \\
\varphi^{\prime}(0) & =M \times\{0\}
\end{aligned}
$$

It is verified in, say, [20, Proposition 3.3], that $\varphi^{\prime}$ is a division of monoids, and we only need to check that it is a division of ordered monoids as well. Let us assume that $m, m^{\prime} \in M, b, b^{\prime} \in B_{Q}^{+}$and $x, x^{\prime} \in C_{c d}^{1}$ are such that $(m, b) \in \varphi^{\prime}(x),\left(m^{\prime}, b^{\prime}\right) \in \varphi^{\prime}\left(x^{\prime}\right)$ and $(m, b) \leq\left(m^{\prime}, b^{\prime}\right)$. Then $m \leq m^{\prime}$ and $b \leq b^{\prime}$. If $b=1$, then $b^{\prime}=b=1$ and $x=x^{\prime}=1$, so that $x \leq x^{\prime}$. If $b=(u, v)$, then $b^{\prime}=b, x=(u, s, v), x^{\prime}=\left(u, s^{\prime}, v\right), m \in \varphi(s)$ and $m^{\prime} \in \varphi\left(s^{\prime}\right)$. Since $\varphi$ is a division of ordered categories and since $s$ and $s^{\prime}$ are coterminal arrows of $C$, it follows that $s \leq s^{\prime}$, and hence $x \leq x^{\prime}$. Finally, if $b=0$, then $x=0$ and hence $x \leq x^{\prime}$.

We can now establish the announced decidability result.

Proposition 1.2 Let $\mathbf{V}$ be a decidable variety of ordered monoids containing $B_{2}^{+}$. Then $\mathbf{g V}$ is decidable.

Proof. Lemma 1.1 shows that if a category $C$ lies in $\mathbf{g V}$, then $C_{c d}^{1} \in \mathbf{V}$. But $C$ divides $C_{c d}^{1}$, so $C \in \mathbf{g V}$ if and only if $C_{c d}^{1} \in \mathbf{V}$. As $C_{c d}^{1}$ is effectively constructible from $C$, it follows that $\mathbf{g} \mathbf{V}$ is decidable.

\subsection{Identities and strongly connected components}

Within the confines of this section, we will consider some non-free infinite categories and semigroupoids. We say that a collection of semigroupoids (resp. categories) of arbitrary cardinality is a Birkhoff variety if it contains 
the one-element semigroup, and is closed under taking divisors and arbitrary direct products and coproducts. As in the case of classes of finite structures, requiring closure under coproduct is not necessary in the definition of a Birkhoff variety of categories.

Adapting the presentation in [20] to the ordered case, we define an identity of ordered categories to be a triple of the form $(X ; x \leq y)$ where $X$ is a graph, and $x$ and $y$ are coterminal paths in $X^{*}$. (Tilson calls the unordered analogue of these identities laws in [20].) We say that an ordered category $C$ satisfies the identity $(X ; x \leq y)$ if for each morphism of categories $\varphi: X^{*} \rightarrow C$, we have $\varphi(x) \leq \varphi(y)$. Identities of ordered semigroupoids are defined in an analogous fashion, considering only triples where $x$ and $y$ have non-zero length.

If $E$ is a set of identities, we denote by $\mathbf{V}(E)$ the class of all ordered semigroupoids (resp. categories) - of arbitrary cardinality -, satisfying the identities in $E$. It is easily verified that $\mathbf{V}(E)$ is a Birkhoff variety, called the Birkhoff variety defined by E. Following step by step the reasoning in [20, Section 9 and Appendix B], we get the following result (see [20, Proposition 9.8] for the unordered case).

Proposition 1.3 Every Birkhoff variety of ordered semigroupoids (resp. categories) is defined by a set of identities.

We say that two sets of identities are equivalent if they define the same Birkhoff variety. A set of identities is trivial if it is satisfied only by trivial semigroupoids.

Example 1.4 Let $Y$ be the graph with two objects $p$ and $q$ and two arrows $a, b: p \rightarrow q$. Then $(Y ; a \leq b)$ is a trivial identity. Indeed, if a semigroupoid $C$ satisfies $(Y ; a \leq b)$ and if $a, b$ are coterminal arrows of $C$, then $a \leq b$ and $b \leq a$, whence $a=b$.

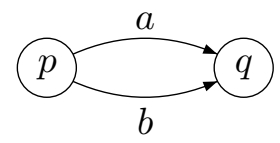

Figure 3: The graph $Y$.

Following Tilson [20] we say that an identity $(X ; x \leq y)$ is a path identity if each arrow of $X$ occurs in $x$ or $y$. As in the unordered case, the rest of this section concerns only categories, and the analogous statements do not hold for semigroupoids. 
Let $X$ be a graph. Two objects $u, v$ of $X$ are strongly connected if $X^{*}(u, v)$ and $X^{*}(v, u)$ are non-empty. This defines an equivalence relation on the set $\operatorname{Obj}(X)$, whose classes are called the strongly connected components of $X$. An arrow $t: u \rightarrow v$ of $X$ is a transition edge if $u$ and $v$ are not in the same strongly connected component. We say that a path identity $(X ; x \leq y)$ is strongly connected if $X$ is a strongly connected graph. We have the following analogue of [20, Theorem 10.2].

Proposition 1.5 Every non-trivial path identity of ordered categories is equivalent to a finite number of strongly connected path identities.

Proof. Let $(X ; x \leq y)$ be a non-trivial path identity of categories. We first assume that $x$ and $y$ traverse the same transition edges $t_{1}, \ldots, t_{r}$. If $r=0$, then $(X ; x \leq y)$ is a strongly connected path identity and we are done. We now assume that $r \geq 1$. Then $x$ and $y$ traverse these transition edges exactly once each, and in the same order: we have factorizations of $x$ and $y$ of the form $x=x_{0} t_{1} x_{1} \cdots t_{r} x_{r}$ and $y=y_{0} t_{1} y_{1} \cdots t_{r} x_{r}$. For each $1 \leq i \leq r$, let $X_{i}$ be the subgraph of $X$ consisting of the vertices and arrows occurring in $u_{i}$ or $v_{i}$. We claim that each $\left(X_{i} ; x_{i} \leq y_{i}\right)$ is a strongly connected path identity and that identity $(X ; x \leq y)$ is equivalent to the collection of strongly connected path identities $\left(\left(X_{0} ; x_{0} \leq y_{0}\right), \ldots,\left(X_{r} ; x_{r} \leq y_{r}\right)\right)$. The proof is exactly the same as that of the analogous case in [20, Theorem 10.2].

Now we assume that there is a transition edge $t: u \rightarrow v$ which occurs in $x$ and not in $y$ (the symmetric case is handled similarly). We need to modify slightly the proof of the analogous case in [20, Theorem 10.2]. Let $n$ and $m$ be the initial and terminal vertices of $x$ (and of $y$ since these two paths are coterminal). Let $Y$ be the graph in Example 1.4 and let $\varphi: X^{*} \rightarrow Y^{*}$ be the morphism defined as follows: if $k$ is a vertex such that there exists a path (possibly empty) in $X$ from $v$ to $k$, then $\varphi(k)=q$; otherwise, $\varphi(k)=p$. If $c: k \rightarrow k^{\prime}$ is an arrow of $X$, we let $\varphi(c)=1_{p}$ if $\varphi(k)=\varphi\left(k^{\prime}\right)=p$, and $\varphi(c)=1_{q}$ if $\varphi(k)=\varphi\left(k^{\prime}\right)=q$. Finally, if $\varphi(k)=p$ and $\varphi\left(k^{\prime}\right)=q$, we let $\varphi(c)=a$ if $c=t$ and $\varphi(c)=b$ otherwise. Note that $\varphi(x)=a$ and $\varphi(y)=b$.

Now any ordered category $C$ satisfying $(X ; x \leq y)$ satisfies $(Y ; \varphi(x) \leq$ $\varphi(y))$, that is, $(Y ; a \leq b)$, and hence $C$ is trivial. Thus $(X ; x \leq y)$ is a trivial path identity, which concludes the proof.

Corollary 1.6 Every non-trivial Birkhoff variety of ordered categories is defined by a set of strongly connected path identities.

We can now prove the ordered analogue of [20, Theorem 11.3]. 
Theorem 1.7 Let $C$ be an ordered category which is not locally trivial. Then $C$ divides a direct product of copies of its strongly connected components. If $C$ is finite, then this product may be assumed to be finite.

Proof. Let $\mathbf{V}$ be the Birkhoff variety generated by the strongly connected components of $C$. By assumption, one of these components is not trivial, so $\mathbf{V}$ is defined by a set of strongly connected path identities by Corollary 1.6. It is easily verified that an ordered category satisfies a strongly connected path identity if and only if each of its strongly connected components does. Thus $C \in \mathbf{V}$, and hence $C$ divides a direct product of copies of its strongly connected components.

If $C$ is finite, each of its strongly connected components is finite, and there are finitely many of them, say, $C_{1}, \ldots, C_{r}$. Let $D=C_{1} \times \cdots \times C_{r}$. Now there exists a division $\delta: C \rightarrow D^{X}$ for some set $X$. For each $x \in X$, let $\delta_{x}: C \rightarrow D$ be the composition of $\delta$ with the projection on the $x$-component of $D^{X}$. If $x, y \in X$ and $\delta_{x}=\delta_{y}$, we let $x \equiv y$. By a classical argument, the relation $\equiv$ is a finite-index equivalence on $X$ and $C$ divides $D^{X / \equiv}$. This concludes the proof.

This theorem is often used in the form of the following corollary.

Corollary 1.8 Let $\mathbf{V}$ be a variety of ordered categories containing $\ell \mathbf{I}$, and let $C$ be a finite ordered category. Then $C \in \mathbf{V}$ if and only if every strongly connected component of $C$ is in $\mathbf{V}$.

\section{Ordered derived semigroupoid}

Our next result is again the ordered version of a theorem due to Tilson [20]. Let $\varphi: S \rightarrow T$ be a relational morphism between semigroups. We define the derived semigroupoid of $\varphi$ as follows. First we define the semigroupoid $R_{\varphi}$ whose objects are the elements of $T^{1}$, and whose arrows from object $t_{1}$ to object $t_{2}$ are the triples of the form $\left(t_{1}, s, t\right)$ where $t \in \varphi(s)$ and $t_{1} t=t_{2}$. Its composition rule is given by $\left(t_{1}, s, t\right)\left(t_{2}, s^{\prime}, t^{\prime}\right)=\left(t_{1}, s s^{\prime}, t t^{\prime}\right)$.

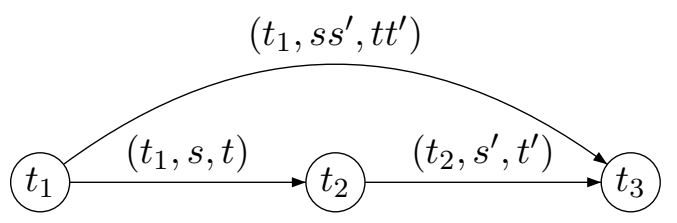

Figure 4: The composition of arrows in $R_{\varphi}$. 
Next we define a relation $\sim$ on $R_{\varphi}$ as follows. If $\left(t_{1}, s, t\right)$ and $\left(t_{1}, s^{\prime}, t^{\prime}\right)$ are coterminal arrows of $R_{\varphi}$, we set

$$
\left(t_{1}, s, t\right) \sim\left(t_{1}, s^{\prime}, t^{\prime}\right) \text { if } s_{1} s=s_{1} s^{\prime} \text { for each } s_{1} \in \varphi^{-1}\left(t_{1}\right) .
$$

By convention and as in [20, Appendix B], if $S$ or $T$ is not a monoid, then $\varphi$ is extended to a relational morphism from $S^{1}$ to $T^{1}$ in the natural way before we compute $\varphi^{-1}(1)$. Now, the relation $\sim$ is a congruence of semigroupoids and the quotient $D_{\varphi}=R_{\varphi} / \sim$ is called the derived semigroupoid of $\varphi$. The $\sim$-class of $\left(t_{1}, s, t\right)$ is written $\left[t_{1}, s, t\right]$.

If $S$ is an ordered semigroup, the derived semigroupoid $D_{\varphi}$ is naturally equipped with the following order relation: if $\left[t_{1}, s, t\right],\left[t_{1}, s^{\prime}, t^{\prime}\right]$ are coterminal arrows of $D_{\varphi}$, we set

$$
\left[t_{1}, s, t\right] \leq\left[t_{1}, s^{\prime}, t^{\prime}\right] \text { if } s_{1} s \leq s_{1} s^{\prime} \text { for each } s_{1} \in \varphi^{-1}\left(t_{1}\right) .
$$

It is immediately verified that $\leq$ is a compatible order on $D_{\varphi}$, and $\left(D_{\varphi}, \leq\right)$ is called the ordered derived semigroupoid of $\varphi$.

We now state the ordered analogue of Tilson's celebrated derived semigroupoid theorem [20, Theorems 16.3 and B.1].

Theorem 2.1 Let $S$ and $V$ be ordered semigroups, and let $T$ be a semigroup.

(1) Let $\theta: S \rightarrow V * T$ be a division, $\pi$ be the natural projection from $V * T$ onto $T$ and let $\varphi=\pi \circ \theta$. Then there exists a division of ordered semigroupoids from $D_{\varphi}$ into $V$.

(2) Let $\varphi: S \rightarrow T$ be a relational morphism such that there exists a division of ordered semigroupoids from $D_{\varphi}$ into $V$. Then $S$ divides the wreath product $V \circ T$ (in the sense of ordered semigroups).

Proof. In order to prove the first statement, we construct a division of ordered semigroupoids from $D_{\varphi}$ into $V$. Our construction is directly adapted of one used by Tilson in an unpublished version of [20]. For each pair $\left(t_{1}, t_{2}\right)$ of elements of $T^{1}$ (i.e. of objects of $D_{\varphi}$ ), and for each element $w$ of $D_{\varphi}\left(t_{1}, t_{2}\right)$, let

$\psi(w)=\left\{t_{1} \cdot v \mid\left[t_{1}, s, t\right]=w\right.$ for some $s \in S, t \in T$ such that $\left.(v, t) \in \theta(s)\right\}$.

It is a routine verification that $\psi$ is a relational morphism from $D_{\varphi}$ in $V$. We now verify that $\psi$ is a division of ordered semigroupoids. Consider in $D_{\varphi}$ two arrows $w$ and $w^{\prime}$ from $t_{1}$ to $t_{2}$. Assume that there exist elements 
$x \in \psi(w)$ and $x^{\prime} \in \psi\left(w^{\prime}\right)$ such that $x \leq x^{\prime}$. Then $x=t_{1} \cdot v$ and $x^{\prime}=t_{1} \cdot v^{\prime}$ for some $v, v^{\prime} \in V$ for which there exist $s, s^{\prime} \in S$ and $t, t^{\prime} \in T$ satisfying the following conditions:

$$
(v, t) \in \theta(s), \quad\left(v^{\prime}, t^{\prime}\right) \in \theta\left(s^{\prime}\right), \quad w=\left[t_{1}, s, t\right], \quad w^{\prime}=\left[t_{1}, s^{\prime}, t^{\prime}\right]
$$

Let $s_{1} \in \varphi^{-1}\left(t_{1}\right)$. Then there exists $v_{1} \in V$ such that $\left(v_{1}, t_{1}\right) \in \theta\left(s_{1}\right)$. Then $\left(v_{1}, t_{1}\right)(v, t)=\left(v_{1}+t_{1} \cdot v, t_{2}\right)$ lies in $\theta\left(s_{1} s\right)$ and $\left(v_{1}, t_{1}\right)\left(v^{\prime}, t^{\prime}\right)=\left(v_{1}+t_{1} \cdot v^{\prime}, t_{2}\right)$ lies in $\theta\left(s_{1} s^{\prime}\right)$. Since $t_{1} \cdot v \leq t_{1} \cdot v^{\prime}$, we have $\left(v_{1}, t_{1}\right)(v, t) \leq\left(v_{1}, t_{1}\right)\left(v^{\prime}, t^{\prime}\right)$. Now, using the fact that $\theta$ is a division of ordered semigroupoids, it follows that $s_{1} s \leq s_{1} s^{\prime}$. As this holds for each $s_{1} \in \varphi^{-1}\left(t_{1}\right)$, we have $w \leq w^{\prime}$. Thus $\psi$ is a division of ordered semigroupoids.

We now prove the second part of the theorem. Let $\psi: D_{\varphi} \rightarrow V$ be a division of ordered semigroupoids. Let $\theta: S \rightarrow V \circ T$ be the relation defined, for each $s \in S$, by

$$
\theta(s)=\left\{(f, t) \in V \circ T \mid t \in \varphi(s), f\left(t_{1}\right) \in \psi\left[t_{1}, s, t\right] \text { for each } t_{1} \in T\right\} .
$$

In the proof of [20, Theorem 5.2], Tilson shows that $\theta$ is a relational morphism, and that it is a division. We need to modify the last part of the proof, to verify that $\theta$ is a division of ordered semigroups. Let $s, s^{\prime} \in S$ be such that there exist $(f, t) \in \theta(s)$ and $\left(f^{\prime}, t^{\prime}\right) \in \theta\left(s^{\prime}\right)$ with $(f, t) \leq\left(f^{\prime}, t^{\prime}\right)$. In particular, we have $t \leq t^{\prime}$, that is, $t=t^{\prime}$. In addition, $f \leq f^{\prime}$, so that $f(1) \leq f^{\prime}(1)$. By definition of $\theta$, we have $f(1) \in \psi[1, s, t]$ and $f^{\prime}(1) \in \psi\left[1, s^{\prime}, t\right]$. Since $\psi$ is a division of ordered semigroupoids, it yields $[1, s, t] \leq\left[1, s^{\prime}, t\right]$. Finally, since $1 \in \varphi^{-1}(1)$, it follows that $s \leq s^{\prime}$. Thus we have proved that $\theta$ is a division of ordered semigroups.

Just like in the unordered case, this result leads to decidability results. For instance, if $S$ is an ordered semigroup, $T$ is a semigroup and $\mathbf{V}$ is a variety of ordered semigroups such that $\mathbf{g V}$ is decidable, then one can decide whether $S$ divides a semidirect product of the form $V * T$, with $V \in \mathbf{V}$. Extending this result by making $T$ a variable, that is, deciding membership in a product variety $\mathbf{V} * \mathbf{W}$ is much more difficult. Standard reasoning on free objects leads to the conclusion that if $\mathbf{V}$ is a variety of ordered semigroups such that $\mathbf{g V}$ is decidable and if $\mathbf{W}$ is a locally finite variety of (unordered) semigroups with effectively computable free objects, then $\mathbf{V} * \mathbf{W}$ is decidable. The following section offers another decidability result for a product variety. 


\section{The delay theorem in the ordered case}

In this section, we state and prove the ordered version of the delay theorem. Let $\mathbf{D}$ be the variety of all finite semigroups $S$ such that $s e=e$ for each $s \in S$ and for each idempotent $e$ of $S ; \mathbf{D}$ is a sub-variety of $\mathbf{L I}$.

For each integer $n \geq 1$, let $\mathbf{D}_{n}$ be the variety of semigroups defined by the identity $y x_{1} \cdots x_{n}=x_{1} \cdots x_{n}$, and let $\mathbf{L} \mathbf{I}_{n}$ be defined by $x_{1} \cdots x_{n} y x_{1} \cdots x_{n}=$ $x_{1} \cdots x_{n}$. It is an elementary verification that $\mathbf{D}_{n} \subseteq \mathbf{L} \mathbf{I}_{n}$ for each $n$, and $\mathbf{D}$ (resp. LI) is the union of the $\mathbf{D}_{n}$ (resp. $\mathbf{L} \mathbf{I}_{n}$ ) for $n \geq 1[2]$.

For each $n \geq 1$, let $A_{n}$ be the set of words on alphabet $A$ of length at most $n$. For each word $w \in A^{+}$, let $\rho_{n}(w)$ be the word equal to $w$ if $|w| \leq n$, and to the length $n$ suffix of $w$ if $|w|>n$. The set $A_{n}$ is made into a semigroup by letting $w \cdot w^{\prime}=\rho_{n}\left(w w^{\prime}\right)$ for each $w, w^{\prime} \in A_{n}$. Then $\rho_{n}$ is a morphism from $A^{+}$onto $A_{n}$. Note that the semigroups $A_{n}$ generate $\mathbf{D}_{n}$.

The delay of a finite semigroup $S$, written $\operatorname{del}(S)$, is defined as follows: if $S$ is a monoid, then $\operatorname{del}(S)=0$; otherwise $\operatorname{del}(S)$ is the least integer $k$ such that either, for each $k$-tuple $\left(s_{1}, \ldots, s_{k}\right)$ of elements of $S$, there exists an idempotent $e$ and an integer $1 \leq i \leq k$ such that $s_{1} \cdots s_{i} e=s_{1} \cdots s_{i}$; or, for each $k$-tuple $\left(s_{1}, \ldots, s_{k}\right)$ of elements of $S$, there exists an idempotent $e$ and an integer $1 \leq i \leq k$ such that $e s_{i} \cdots s_{k}=s_{i} \cdots s_{k}$. It is easily verified that if $S^{n}=S^{n+1}$, then $n \geq \operatorname{del}(S)$. In particular, $|S| \geq \operatorname{del}(S)$ (for instance $[20])$.

Finally, the Cauchy category of $S$, denoted $S_{E}$, has object set the set of idempotents of $S$, and its arrow sets are of the form $S_{E}(e, f)=e S f$ for all $e, f \in E$. If $S$ is ordered, then each set $e S f$ is ordered as well, and this makes $S_{E}$ an ordered category.

Now we prove the following result, the ordered counterpart of the Delay theorem [20, Theorem 17.1].

Theorem 3.1 If $\mathbf{V}$ is a variety of ordered monoids, then $\mathbf{V} * \mathbf{D}=\mathbf{V} * \mathbf{L I}$. An ordered semigroup $S$ belongs to $\mathbf{V} * \mathbf{L I}$ if and only if $S_{E} \in \mathbf{g V}$. Moreover, if $n \geq \operatorname{del}(S)$, then $S \in \mathbf{V} * \mathbf{L I}$ if and only if $S \in \mathbf{V} * \mathbf{D}_{n}$.

Before we prove Theorem 3.1, let us note a few of its consequences.

One verifies immediately that if $\mathbf{V}$ is a variety of ordered monoids and if $S$ is an ordered semigroup, then $S \in \mathbf{L V}$ if and only if $S_{E} \in \ell \mathbf{V}$. In view of the Delay theorem, this yields the following corollary.

Corollary 3.2 Let $\mathbf{V}$ be a variety of ordered monoids. Then $\mathbf{V}$ is local if and only if $\mathbf{L V}=\mathbf{V} * \mathbf{L I}$. 
Proof. If $\mathbf{V}$ is local and $S$ is an ordered semigroup, then we have

$$
S \in \mathbf{L V} \Longleftrightarrow S_{E} \in \ell \mathbf{V} \Longleftrightarrow S_{E} \in \mathbf{g V} \Longleftrightarrow S \in \mathbf{V} * \mathbf{L I},
$$

so that $\mathbf{L V}=\mathbf{V} * \mathbf{L I}$.

To verify the converse, we first note that if $C$ is an ordered category, then $C$ and $\left(C_{c d}\right)_{E}$ divide each other as ordered categories. The proof in [20, Proposition 16.1] of the unordered version of this result carries over to the ordered case without any difficulty.

Let us now assume that $\mathbf{L V}=\mathbf{V} * \mathbf{L I}$. If $C$ is an ordered category in $\ell \mathbf{V}$, the ordered category $\left(C_{c d}\right)_{E}$ also lies in $\ell \mathbf{V}$. It follows that $C_{c d} \in \mathbf{L V}$, so $C_{c d} \in \mathbf{V} * \mathbf{L I}$ and hence $\left(C_{c d}\right)_{E} \in \mathbf{g V}$. But $C$ divides $\left(C_{c d}\right)_{E}$, so $C \in \mathbf{g V}$ as expected.

Of course, if $\mathbf{V}$ is local and decidable, it follows that $\mathbf{V} * \mathbf{L I}$ is decidable. More generally, the following result is immediately deduced from Theorem 3.1.

Corollary 3.3 Let $\mathbf{V}$ be a variety of ordered monoids. If $\mathbf{g V}$ is decidable, then $\mathbf{V} * \mathbf{L I}$ is decidable.

In view of Proposition 1.2, we have the following particular case.

Corollary 3.4 Let $\mathbf{V}$ be a decidable variety of ordered monoids containing $B_{2}^{+}$. Then $\mathbf{V} * \mathbf{L I}$ is decidable.

We also note the following refinement of the equality $\mathbf{V} * \mathbf{D}=\mathbf{V} * \mathbf{L I}$, which is useful in the context of applications to formal language theory (see [11, Proposition 4.22]).

Proposition 3.5 Let $\mathbf{V}$ be a non-trivial variety of ordered monoids and let $n \geq 1$ be an integer. Then $\mathbf{V} * \mathbf{L} \mathbf{I}_{n}=\mathbf{V} * \mathbf{D}_{n}$.

Proof. To prove the non-trivial containment, let us consider a generator of the variety $\mathbf{V} * \mathbf{L} \mathbf{I}_{n}$ in the form of a unitary semidirect product $V * T$ with $V \in \mathbf{V}$ and $T \in \mathbf{L} \mathbf{I}_{n}$. One verifies immediately that if $\left(v_{1}, t_{1}\right), \ldots,\left(v_{n}, t_{n}\right)$ are elements of $V * T$, then $\left(1, t_{1} \cdots t_{n}\right)$ is idempotent and

$$
\left(v_{1}, t_{1}\right) \cdots\left(v_{n}, t_{n}\right)\left(1, t_{1} \cdots t_{n}\right)=\left(v_{1}, t_{1}\right) \cdots\left(v_{n}, t_{n}\right),
$$

so $\operatorname{del}(V * T) \leq n$. Now Theorem 3.1 implies $V * T \in \mathbf{V} * \mathbf{D}_{n}$, as expected. 
Finally, we prove two propositions, Propositions 3.6 and 3.8, which (together with the ordered derived semigroupoid theorem, Theorem 2.1 above) establish Theorem 3.1.

Proposition 3.6 Let $S$ be an ordered semigroup, let $n \geq|S|$, and let $\sigma: A^{+} \rightarrow S$ be an onto morphism. Let $\varphi: S \rightarrow A_{n}$ be the relational morphism given by $\varphi=\rho_{n} \circ \sigma^{-1}$. Then $D_{\varphi}$ divides an element of the variety of ordered categories generated by $S_{E}$.

Proof. Here again, we rely on the proof of the analogous statement in [20] (see the proof of Theorem 18.2 therein). We first consider the ordered monoid $S^{1}$, the ordered monoid $A_{n}^{1}$ and $\psi$, the natural extension of $\varphi$ to a relational morphism $\psi: S^{1} \rightarrow A_{n}^{1}$. Then $D_{\psi}$ is an ordered category and $D_{\varphi}$ divides $D_{\psi}$ as an ordered semigroupoid.

Next we show that $D_{\psi}$ lies in the variety generated by $S_{E}$. Using Theorem 1.7, it suffices to consider the only non-trivial bonded component of $D_{\psi}$, namely the subcategory of $D_{\psi}$ whose set of vertices is $A^{n}$, the set of words of length $n$. We assume that for each word $w$ of length $n$, there exist an idempotent $e$ of $S$ and a factorization $w=u v$ of $w$ such that $\sigma(u)=\sigma(u) e$. (By definition of $\operatorname{del}(S)$, if that is not the case, then for each word $w$ of length $n$, there exist an idempotent $e$ of $S$ and a factorization $w=u v$ of $w$ such that $\sigma(v)=e \sigma(v)$ and the proof is dual of what follows.) One fixes $u_{w}$ to be the shortest such word $u$, setting $v_{w}$ to be the word such that $w=u_{w} v_{w}$, and one lets $\theta(w)=e_{w}$ be an idempotent such that $\sigma\left(u_{w}\right)=\sigma\left(u_{w}\right) e_{w}$.

Let $w_{1}, w_{2}$ be words of length $n$. If $x \in D_{\psi}\left(w_{1}, w_{2}\right)$, let $\theta(x)$ be the following subset of $S_{E}\left(e_{w_{1}}, e_{w_{2}}\right)$ :

$$
\begin{aligned}
\theta(x)= & \left\{t \in e_{w_{1}} S e_{w_{2}} \mid \sigma\left(u_{w_{1}}\right) t \sigma\left(v_{w_{2}}\right)=\sigma\left(w_{1}\right) s \text { and } \sigma\left(u_{w_{1}}\right) t \leq_{\mathcal{L}} \sigma\left(u_{w_{2}}\right)\right. \\
& \text { for some } \left.s, w \text { such that }\left[w_{1}, s, w\right]=x\right\} .
\end{aligned}
$$

In the proof of [20, Theorem 18.2], it is shown that $\theta$ is a relational morphism and a division of categories.

Let us verify that $\theta$ is also a division of ordered categories. Let $x, x^{\prime} \in$ $D_{\psi}\left(w_{1}, w_{2}\right)$ and let $t \in \theta(x)$ and $t^{\prime} \in \theta\left(x^{\prime}\right)$ be such that $t \leq t^{\prime}$. Then there exist elements $s, s^{\prime} \in S^{1}$ and words $w, w^{\prime}$ such that

$$
\begin{array}{rrrl}
{\left[w_{1}, s, w\right]} & =x & \sigma\left(u_{w_{1}}\right) t \sigma\left(v_{w_{2}}\right) & =\sigma\left(w_{1}\right) s \\
{\left[w_{1}, s^{\prime}, w^{\prime}\right]} & =x^{\prime} & \sigma\left(u_{w_{1}}\right) t^{\prime} \sigma\left(v_{w_{2}}\right) & =\sigma\left(w_{1}\right) s^{\prime} \\
\rho_{n}\left(w_{1} w\right)=\rho_{n}\left(w_{1} w^{\prime}\right) & =w_{2}
\end{array}
$$

In particular, we have

$$
\sigma\left(w_{1}\right) s=\sigma\left(u_{w_{1}}\right) t \sigma\left(v_{w_{2}}\right) \leq \sigma\left(u_{w_{1}}\right) t^{\prime} \sigma\left(v_{w_{2}}\right)=\sigma\left(w_{1}\right) s^{\prime} .
$$


Now let $s_{1} \in \psi^{-1}\left(w_{1}\right)$. Then there exists a word $x_{1}$ such that $\sigma\left(x_{1} w_{1}\right)=s_{1}$. By left multiplication of the above inequality by $\sigma\left(x_{1}\right)$, we get $s_{1} s \leq s_{1} s^{\prime}$. It follows that

$$
x=\left[w_{1}, s, w\right] \leq\left[w_{1}, s^{\prime}, w^{\prime}\right]=x^{\prime},
$$

thus proving that $\theta$ is a division of ordered categories.

The next result is the ordered analogue of [20, Lemma 18.3].

Lemma 3.7 Let $S$ and $T$ be ordered semigroups. If $S$ divides $T$ in the sense of ordered semigroups, then $S_{E}$ divides $T_{E}$ in the sense of ordered categories.

Proof. Let $\varphi: S \rightarrow T$ be a division. Since $T$ is finite, for each idempotent $e$ of $S, \varphi(e)$ is a non-empty semigroup, so we can choose an idempotent $\psi(e)$ in $\varphi(e)$. Next, for each pair $\left(e, e^{\prime}\right)$ of objects of $S_{E}$, let $\psi$ be the relation from $S_{E}\left(e, e^{\prime}\right)$ into $T_{E}\left(\psi(e), \psi\left(e^{\prime}\right)\right)$ given by $\psi(s)=\psi(e) \varphi(s) \psi\left(e^{\prime}\right)$. In the proof of [20, Lemma 18.3], Tilson proves that $\psi$ is a relational morphism, and a division of categories. We now verify that it is also a division of ordered categories.

Let $s, s^{\prime} \in S_{E}\left(e, e^{\prime}\right)$, and let us assume that $t \leq t^{\prime}$ for some $t \in \psi(s)$ and $t^{\prime} \in \psi\left(s^{\prime}\right)$. By definition of $\psi$, there exist $x \in \varphi(s)$ and $x^{\prime} \in \varphi\left(s^{\prime}\right)$ such that $\psi(e) x \psi\left(e^{\prime}\right) \leq \psi(e) x^{\prime} \psi\left(e^{\prime}\right)$. As $\psi(e) \in \varphi(e)$, we have $\psi(e) x \psi\left(e^{\prime}\right) \in \varphi\left(e s e^{\prime}\right)=$ $\varphi(s)$. Similarly, $\psi(e) x^{\prime} \psi\left(e^{\prime}\right) \in \varphi\left(s^{\prime}\right)$. Since $\varphi$ is a division, it follows that $s \leq s^{\prime}$, which concludes the proof.

Now we can prove the following.

Proposition 3.8 Let $\mathbf{V}$ be a variety of ordered monoids and let $S \in \mathbf{V} * \mathbf{L I}$. Then $S_{E}$ lies in $\mathbf{g V}$.

Proof. We proceed as in the proof of the analogous, unordered result, Proposition 18.4 in [20]. Let $S \in \mathbf{V} * \mathbf{L I}$ : there exist elements $T \in \mathbf{V}$ and $W \in \mathbf{L I}$ such that $S$ divides $T * W$. In view of the above lemma, it suffices to show the proposition when $S=T * W$.

Let $(f, e)$ and $\left(f^{\prime}, e^{\prime}\right)$ be idempotent elements of $S=T * W$. Let $E$ be the set of idempotents of $S$. Let $\varphi: S_{E}\left((f, e),\left(f^{\prime}, e^{\prime}\right)\right) \rightarrow T^{E}$ be the mapping such that $\varphi(t, w): E \rightarrow T$ maps any idempotent $g$ of $S$ to get. In the proof of [20, Proposition 18.4], Tilson shows that $\varphi$ is a relational morphism from $S_{E}$ to $T^{E}$ and that it is a division of categories. Again we verify that it is a division of ordered categories. Let us assume that $(t, w)$ and $\left(t^{\prime}, w^{\prime}\right)$ are elements of $S_{E}\left((f, e),\left(f^{\prime}, e^{\prime}\right)\right)$ such that $\varphi(t, w) \leq \varphi\left(t^{\prime}, w^{\prime}\right)$. That is, for each idempotent $g$ of $S$, we have get $\leq$ get $^{\prime}$. Since $(f, e)(t, w)\left(f^{\prime}, e^{\prime}\right)=(t, w)$, we 
have $e w e^{\prime}=w$ : in a semigroup of LI, this implies that $w=e e^{\prime}$. Similarly $w^{\prime}=e e^{\prime}$, so $w=w^{\prime}$. Also we have $t=f+e t+e w f^{\prime}=f+e t+e e^{\prime} f^{\prime}$. Since get $\leq$ get $t^{\prime}$ for each idempotent $g$, letting $g=e$ we get $e t \leq e t^{\prime}$. As a result, we have $t=f+e t+e e^{\prime} f^{\prime} \leq f+e t^{\prime}+e e^{\prime} f^{\prime}=t^{\prime}$. Thus $(t, w) \leq\left(t^{\prime}, w^{\prime}\right)$, which concludes the proof.

\section{Locality of certain varieties}

In this section, we prove that $\mathbf{J}_{1}^{+}$is local, like its unordered counterpart $\mathbf{J}_{1}$. We also prove that $\mathbf{J}^{+}$is local. This result is more surprising, since $\mathbf{J}$ is the canonical example of a non-local variety.

\subsection{Locality of $\mathrm{J}_{1}^{+}$}

Let $C$ be a category. If $x$ is an arrow in the free category $C^{*}$ (that is, a path in the graph $C$ or equivalently a finite sequence of consecutive arrows of $C$ ), we denote by $[x]_{C}$ (or $[x]$ if $C$ is understood) the evaluation of $x$ in $C$, that is, the product in $C$ of the arrows constituting $x$. The content $c(x)$ of $x$ is the set of arrows of $C$ occurring in $x$.

If $x, y$ are arrows in $C^{*}$, we write $x \leq y$ if $x$ and $y$ are coterminal and if $c(y) \subseteq c(x)$. This relation is easily seen to be a compatible quasi-order on $C^{*}$.

We need the following technical result [2, Lemma 7.3].

Lemma 4.1 Let $C$ be an ordered category in $\ell \mathbf{J}_{1}^{+}$. Let $u, v$ be objects of $C$, and let $x \in C^{*}(u, v)$ and $y \in C^{*}(v, v)$ be consecutive paths in $C$. If $c(y) \subseteq c(x)$, then $[x y]=[x]$.

The proof of the following proposition is adapted from the proof of a result due to Simon in the non-ordered case, see [2, Theorem 7.1].

Proposition 4.2 Let $C$ be an ordered category. Then $C \in \ell \mathbf{J}_{1}^{+}$if and only if, whenever $x, y$ are coterminal paths in $C, x \leq y$ implies $[x] \leq[y]$.

Proof. Let us assume that $x \leq y$ implies $[x] \leq[y]$ whenever $x, y$ are coterminal paths in $C$. It is immediate that if $x, y$ are loops around some object $u$, then $x \leq 1_{u}, x x \leq x \leq x x$ and $x y \leq y x$. It follows that $[x] \leq 1_{u}$, $[x]^{2}=[x]$ and $[x y]=[y x]$ in $C$. In particular, $C \in \ell \mathbf{J}_{1}^{+}$.

To prove the converse implication, assume that $C \in \ell \mathbf{J}_{1}^{+}$and consider coterminal paths $x, y \in C^{*}(u, v)$ such that $x \leq y$. We show that $[x] \leq[y]$ 
by induction on the cardinality of $c(x)$. If $c(x)=\emptyset$, the result is trivial. We now assume that $c(x) \neq \emptyset$, and we let $X$ be the subgraph of $C$ consisting of the arrows in $c(x)$.

If $X$ is strongly connected, then there exists a path $z: v \rightarrow u$ such that $c(z) \subseteq c(x)$. Then $z y \in C^{*}(v, v)$ and we have $[x]=[x z y]$ by Lemma 4.1. But $x z$ is also a loop, so we have $[x]=[(x z) y]=[x z][y] \leq[y]$ as expected.

If $X$ is not strongly connected, then $v$ and $u$ are not in the same strongly connected component of $X$. Thus there exists a transition edge $a: s \rightarrow t$ which occurs in $y$, and $y$ factors as $y=y_{0} a y_{1}$. Since $c(y)$ is contained in $c(x)$, there also exists a factorization $x=x_{0} a x_{1}$. As $a$ is a transition edge, these factorizations are unique and $c\left(y_{0}\right) \subseteq c\left(x_{0}\right)$ and $c\left(y_{1}\right) \subseteq c\left(x_{1}\right)$. In addition, since $a$ is a transition edge, it occurs exactly once in $x$. In particular $c\left(x_{0}\right)$ and $c\left(x_{1}\right)$ are proper subsets of $c(x)$. By induction, it follows that $\left[x_{0}\right] \leq\left[y_{0}\right]$ and $\left[x_{1}\right] \leq\left[y_{1}\right]$, and hence $[x] \leq[y]$.

We can now prove the announced result.

Theorem 4.3 The variety $\mathbf{J}_{1}^{+}$is local.

Proof. Let $C$ be a category in $\ell \mathbf{J}_{1}^{+}$and let $A$ be the set of all arrows of $C$. The set $\mathcal{P}(A)$ of subsets of $A$, equipped with the operation of union and the reverse inclusion relation is easily seen to be an ordered monoid in $\mathbf{J}_{1}^{+}$. In order to prove the theorem, it suffices to show that the content relation $\kappa: C \rightarrow \mathcal{P}(A)$ given by $\kappa(x)=\left\{c\left(x^{\prime}\right) \mid x^{\prime} \in C^{*},\left[x^{\prime}\right]=x\right\}$, defines a division of ordered categories.

The multiplicativity condition in the definition of a division is immediately verified. Now let $x, y$ be coterminal arrows of $C$ and let us assume that there exist paths $x^{\prime}$ and $y^{\prime}$ in $C$ such that $\left[x^{\prime}\right]=x,\left[y^{\prime}\right]=y$ and $c\left(x^{\prime}\right) \supseteq c\left(y^{\prime}\right)$, that is, $x \leq y$. By Proposition 4.2 it follows that $\left[x^{\prime}\right] \leq\left[y^{\prime}\right]$, i.e. $[x] \leq[y]$. This concludes the proof.

In view of Corollary 3.2, we have now:

Corollary 4.4 $\mathbf{L} \mathbf{J}_{1}^{+}=\mathbf{J}_{1}^{+} * \mathbf{D}$.

\section{$4.2 \quad$ Locality of $\mathrm{J}^{+}$}

\subsubsection{Some combinatorics on paths}

Let $C$ be a graph and let $m \geq 1$ be an integer. For each arrow $x$ of $C^{*}$, i.e. for each path $x$ in $C$, we define $c_{m}(x)$ to be the set of tuples $\left(a_{1}, \ldots, a_{m}\right)$ of 
arrows of $C$ such that $x$ can be factored as $x=x_{0} a_{1} x_{1} \cdots a_{m} x_{m}$. Note that $c_{1}(x)$ is the content of $x$ defined in the previous section.

We define a relation $\leq_{m}$ on $C^{*}$ by letting $x \leq_{m} y$ if $x$ and $y$ are coterminal and $c_{r}(y) \subseteq c_{r}(x)$ for each $r \leq m$. The relation $\leq_{m}$ is a compatible quasiorder on $C^{*}$. What happens if $c_{r}(y) \subseteq c_{r}(x)$ for each $r \leq m$ but $x$ and $y$ are not coterminal, is described in the following statement, which is related with [18, Lemma 2.2].

Proposition 4.5 Let $C$ be a graph, let $u, v, s, t$ be objects of $C$ and let $x$ be a path from $u$ to $v$ and $y$ a path from $s$ to $t$. If $c_{r}(y) \subseteq c_{r}(x)$ for each $r \leq m$, then there exist paths $x_{0}$ from $u$ to $s$ and $x_{1}$ from $t$ to $v$ such that $x \leq_{m} x_{0} y x_{1}$.

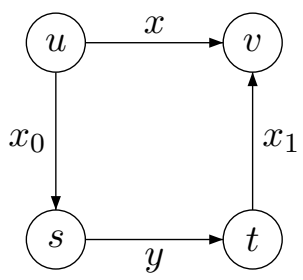

Proof. Let $x_{0}$ be the shortest prefix of the path $x$ which ends in $s$ : such a prefix exists since the first arrow of $y$ is guaranteed to occur in $x$. Let $x^{\prime}$ be the corresponding suffix of $x$, that is, $x=x_{0} x^{\prime}$.

Let $r \leq m$. We first claim that $c_{r}\left(x_{0} y\right) \subseteq c_{r}(x)$. Indeed let $z \in c_{r}\left(x_{0} y\right)$. Then the tuple $z$ is the concatenation of a tuple $z_{0} \in c_{n}\left(x_{0}\right)$ and a tuple $z^{\prime} \in c_{r-n}(y)$ for some $0 \leq n \leq r,-$ which we write $z=z_{0} z^{\prime}$. If $n=0$, then $z=z^{\prime} \in c_{r}(y)$, and hence $z \in c_{r}(x)$. We now assume that $n>0$. Since $z^{\prime} \in c_{r-n}(y)$, we have $z^{\prime} \in c_{r-n}(x)$. If the first arrow of $y$, say $a$, is also the first arrow of $z^{\prime}$, then $z^{\prime}$ is in fact in $c_{r-n}\left(x^{\prime}\right)$ by definition of $x_{0}$ and $x^{\prime}$. Thus $z=z_{0} z^{\prime} \in c_{r}\left(x_{0} x^{\prime}\right)=c_{r}(x)$. Otherwise, $a z^{\prime} \in c_{r-n+1}(y)$, so $a z^{\prime} \in c_{r-n+1}(x)$ and, as above, $a z^{\prime} \in c_{r-n+1}\left(x^{\prime}\right)$. It follows that $z^{\prime} \in c_{r-n}\left(x^{\prime}\right)$ and hence $z=z_{0} z^{\prime} \in c_{r}\left(x_{0} x^{\prime}\right)=c_{r}(x)$.

By the left-right dual of this reasoning applied to the paths $x$ and $x_{0} y$, there also exists a suffix $x_{1}$ of $x$ starting in $v$ such that $c_{r}\left(x_{0} y x_{1}\right) \subseteq c_{r}(x)$. This concludes the proof.

A 2-factorization of a path $x$ in $C$ is a triple of the form $\left(x_{0}, a, x_{1}\right)$ such that $x_{0}$ and $x_{1}$ are arrows of $C^{*}, a$ is either an arrow of $C$ or an empty path in $C^{*}$, and $x=x_{0} a x_{1}$ in $C^{*}$. If $x, y$ are paths in $C$, we let $x \leq_{m}^{2} y$ if for every 2 -factorization $\left(x_{0}, a, x_{1}\right)$ of $x$, there exists a 2 -factorization $\left(y_{0}, b, y_{1}\right)$ of $y$ such that $c_{r}\left(y_{0}\right) \subseteq c_{r}\left(x_{0}\right)$ and $c_{r}\left(y_{1}\right) \subseteq c_{r}\left(x_{1}\right)$ for each $r \leq m$, and $b=a$ 
or $b$ is an empty path of $C^{*}$. The following result is proved in [18, Theorem 2.3]. (In fact, [18] states a symmetrized version of this result, but the proof is exactly that of our statement.)

Lemma 4.6 Let $C$ be a category, let $x, y$ be arrows of $C^{*}$ and let $m \geq 1$ be an integer. Then $x \leq_{m}^{2} y$ if and only if $c_{r}(y) \subseteq c_{r}(x)$ for each $r \leq 2 m$.

\subsubsection{Categories locally in $\mathrm{J}^{+}$}

Let $\mathbf{V}_{m}$ be the class of all ordered categories $C$ such that if $x, y$ are arrows of $C^{*}$ such that $x \leq_{m} y$, then $x \leq y$. Let $\mathbf{V}$ be the union of the $\mathbf{V}_{m}(m \geq 1)$.

Lemma 4.7 The classes $\mathbf{V}$ and $\mathbf{V}_{m}(m \geq 1)$ are varieties of ordered categories.

Proof. Let $C$ be an ordered category in $\mathbf{V}_{m}$ and let $D$ be an ordered category. Let $\varphi: D \rightarrow C$ be an ordered-faithful morphism. Then $\varphi$ extends to a length-preserving morphism $\varphi: D^{*} \rightarrow C^{*}$, such that $[\varphi(x)]_{C}=\varphi\left([x]_{D}\right)$ for each arrow $x$ of $D^{*}$. Let $x, y$ be arrows of $D^{*}$ such that $x \leq_{m} y$. It is immediately verified that the paths $\varphi(x)$ and $\varphi(y)$ in $C$ satisfy $\varphi(x) \leq_{m} \varphi(y)$. Therefore $[\varphi(x)]_{C} \leq[\varphi(y)]_{C}$ and hence, $[x]_{D} \leq[y]_{D}$ since $\varphi$ is orderedfaithful.

Now let $\varphi: C \rightarrow D$ be a quotient morphism of ordered categories. By definition of a quotient morphism, we may assume that $C$ and $D$ have the same set of objects. For each arrow $a: u \rightarrow v$ of $D$ we fix an arrow $\psi(a): u \rightarrow$ $v$ in $C$ such that $\varphi(\psi(a))=a$. This choice induces a length-preserving morphism $\psi: D^{*} \rightarrow C^{*}$ such that $\varphi\left([\psi(x)]_{C}\right)=[x]_{D}$ for each arrow $x$ of $D^{*}$. Let $x, y$ be arrows of $D^{*}$ such that $x \leq_{m} y$. Then $\psi(x) \leq_{m} \psi(y)$ in $C^{*}$, and hence $[\psi(x)]_{C} \leq[\psi(y)]_{C}$. It follows that $[x]_{D} \leq[y]_{D}$ since $\varphi$ is a morphism of ordered categories.

Thus $\mathbf{V}_{m}$ is closed under taking inverse images by ordered-faithful morphisms and direct images by quotient morphisms of ordered categories. That is, $\mathbf{V}_{m}$ is closed under division. The verification that $\mathbf{V}_{m}$ is closed under taking direct products is routine. So $\mathbf{V}_{m}$ is a variety for any $m$. It is immediate that $\mathbf{V}_{m}$ is contained in $\mathbf{V}_{m+1}$ for each $m$ : it follows easily that $\mathbf{V}$ is a variety as well.

Our next result is the following theorem, analogous to results of Knast [5] and Thérien [18] on gJ and to Theorem 4.2 above.

Theorem $4.8 \ell \mathbf{J}^{+}=\mathrm{V}$. 
Before we can prove Theorem 4.8, we need to introduce some notation, borrowed from [20,18]. If $C$ is a category, an ideal is a subset $J$ of $C$ such that $J=C J C$. If $x$ and $y$ are arrows of $C$, we let $x \leq \mathcal{J} y$ if $x$ lies in the ideal generated by $y$, that is, if $x \in C y C$. We write $x \mathcal{J} y$ if $x \leq \mathcal{J} y$ and $y \leq_{\mathcal{J}} x$ : then $\mathcal{J}$ is an equivalence relation on the arrows of $X$. We say that $I$ is a minimal ideal of $C$ if $I$ is a non-empty ideal of $C$ and if it does not contain properly a non-empty ideal of $C$. It is easily verified [18, Lemma 1.1] that if $C$ is strongly connected, then $C$ has a unique minimal ideal $I$ and that $I(u, v) \neq \emptyset$ for any pair $(u, v)$ of objects of $C$.

If the minimal ideal $I$ of $C$ is trivial, a 0 -minimal ideal of $C$ is an ideal $K$ such that the only non-empty ideal properly contained in $K$ is $I$ itself. It can be shown [18, Lemma 1.2] that in that case, any 0 -minimal ideal is of the form $I \cup J$, where $J$ is a $\mathcal{J}$-class of $C$. Moreover, $C$ divides a direct product of the form $C_{1} \times \cdots \times C_{r}$, where each $C_{i}$ is a divisor of $C$ with a unique minimal ideal which is trivial, and with a unique 0 -minimal ideal.

\section{Proof of Theorem 4.8.}

Let $C$ be an ordered category. If $C \in \mathbf{V}$, then $C \in \mathbf{V}_{m}$ for some $m \geq 1$. It is immediate that if $x$ is a loop of $C^{*}$ around some objet $u$, then $x \leq_{m} 1_{u}$, so $[x]_{C} \leq 1_{u}$ for any $x \in C(u, u)$. Thus $\mathbf{V} \subseteq \ell \mathbf{J}^{+}$.

We now assume that $C \in \ell \mathbf{J}^{+}$. We show that $C \in \mathbf{V}$ by induction on the cardinality of the set of arrows of $C$. The result is trivial if $C$ has zero or one arrow, or more generally if $C$ is a trivial category. Let us consider the case where $C$ has at least two arrows. Since $\mathbf{V}$ is a variety and in view of Theorem 1.7, we may assume without loss of generality that $C$ is strongly connected. According to the above discussion, $C$ has a unique minimal ideal.

Let us verify that this minimal ideal, say $I$, is trivial. For each object $u$, $C(u, u) \in \mathbf{J}^{+}$, so $C(u, u)$ has a zero, say $0_{u}$. Then the set

$$
\bigcup\left\{0_{u} x 0_{v} \mid x \in I(u, v)\right\}
$$

(where the union runs over all pairs $(u, v)$ of objects of $C$ ) is easily checked to be an ideal contained in $I$. As $I$ is minimal, it follows that $x=0_{u} x 0_{v}$ for each $x \in I(u, v)$ and in particular, $I(u, u)=0_{u}$ for each object $u$. Now let $x, y \in I(u, v)$. Since $C$ is strongly connected, we may consider an element $z \in C(v, u)$. Then $x z, y z \in I(u, u)$, so $x z=y z=0_{u}$. Similarly, $z x=z y=$ $0_{v}$. Finally, $x=0_{u} x=y z x=y 0_{v}=y$.

Thus the minimal ideal $I$ of $C$ is trivial and, according to the above discussion, we may assume, again without loss of generality, that $C$ also has a unique 0 -minimal ideal, of the form $I \cup J$ where $J$ is a $\mathcal{J}$-class. 
Observe that $C \in \ell \mathbf{J}^{+}$implies the following: if $x, y \in C(u, v)$ are coterminal arrows of $C$ and if $x \leq \mathcal{J} y$, then $x=x_{0} y x_{1}$ where $x_{0} \in C(u, u)$ and $x_{1} \in C(v, v)$ are loops. It follows that $x \leq 1_{u} y 1_{v}=y$. In particular, if $x, y \in C(u, v)$ and $x \in I$, then $x \leq y$.

We first assume that $J$ contains an idempotent $e$. Then $C \backslash I$ is a proper subcategory of $C$. Indeed, let $s, t$ be consecutive arrows in $C \backslash I$. By definition of $J$, we have $e \leq_{\mathcal{J}} s$ and $e \leq_{\mathcal{J}} t$, so $e=s_{0} s s_{1}=t_{0} t t_{1}$. Now $e=e e=s_{0} s s_{1} t_{0} t t_{1}$. Since $s$ and $t$ are assumed to be consecutive, $s_{1} t_{0} \in C(u, u)$ for some object $u$, so $s_{1} t_{0} \leq 1_{u}$ and $e \leq s_{0} s t t_{1}$. If $s t \in I$, then $s_{0} s t t_{1} \in I$, so $s_{0} s t t_{1} \leq \mathcal{J} e$, whence $s_{0} s t t_{1} \leq e$, and finally $s_{0} s t t_{1}=e$. It follows that $e \in I$, a contradiction.

Thus in that case, $C \backslash I \in \ell \mathbf{J}^{+}$and, by induction, there exists an integer $m$ such that $x \leq_{m} y$ in $(C \backslash I)^{*}$ implies $[x]_{C \backslash I} \leq[y]_{C \backslash I}$. Now let $x, y$ be coterminal arrows in $C^{*}$ such that $x \leq_{m} y$. If $x \in(C \backslash I)^{*}$, then $y \in(C \backslash I)^{*}$ and $x \leq_{m} y$ in $(C \backslash I)^{*}$, so we have $[x]_{C \backslash I} \leq[y]_{C \backslash I}$. Since $C \backslash I$ is a subcategory of $C$, we have $[x]_{C \backslash I}=[x]_{C}$ and $[y]_{C \backslash I}=[y]_{C}$, and the expected inequality follows. If $x \notin(C \backslash I)^{*}$, then $[x]_{C} \in I$. As observed above, this implies that $[x]_{C} \leq[y]_{C}$.

Now we assume that $J$ does not contain an idempotent. Then we have $J J \subseteq I$. Otherwise there exist consecutive arrows $x, y$ in $J$ such that $x y \in J$. Then $x \leq_{\mathcal{J}} x y$, so $x=s x y t$ and hence, $x=s^{k} x(y t)^{k}$ for any integer $k \geq 1$. Since $C$ is finite, some power $(y t)^{k}$ is idempotent, it lies in $I \cup J$, and it does not lie in $I$ since $x \notin I$. Thus $(y t)^{k} \in J$, a contradiction.

Let $D$ be the quotient of $C$ by the ideal $I \cup J$ : as an ordered set, $D$ is equal to $C \backslash J$ and the product in $D$ is the following. Let $x, y$ be consecutive arrows in $C \backslash J$. If the product $x y$ in $C$ is not in $J$, then the product $x \cdot y$ in $D$ is set to be $x \cdot y=x y$. If in $C$ we have $x y \in J$, then in $D$ we let $x \cdot y$ be the unique arrow of $I$ which is coterminal with $x y$. The category $D$ is immediately verified to be a quotient of $C$ so $D \in \ell \mathbf{J}^{+}$. By induction, there exists an integer $m$ such that, if $x, y$ are coterminal arrows in $D$ such that $x \leq_{m} y$, then $[x]_{D} \leq[y]_{D}$.

Let $x, y$ be coterminal arrows in $C^{*}$ such that $x \leq_{2 m} y$. We want to show that $[x]_{C} \leq[y]_{C}$. By Lemma 4.6, we have $x \leq_{m}^{2} y$.

Let us first assume that $x$ admits a factorization $x=x_{0} x_{1}$ such that $\left[x_{0}\right]_{C},\left[x_{1}\right]_{C} \notin I \cup J$. Since $x \leq_{m}^{2} y, y$ can be factored as $y=y_{0} y_{1}$ with $c_{r}\left(y_{0}\right) \subseteq c_{r}\left(x_{0}\right)$ and $c_{r}\left(y_{1}\right) \subseteq c_{r}\left(x_{1}\right)$ for each $r \leq m$. By Proposition 4.5 there exist paths $s, t, s^{\prime}, t^{\prime}$ such that $x_{0} \leq_{m} s y_{0} t$ and $x_{1} \leq_{m} s^{\prime} y_{1} t^{\prime}$. It follows that $x_{0}, x_{1}, y_{0}, y_{1}, s, t, s^{\prime}, t^{\prime}$ are paths in $D^{*}$, and that

$$
\left[x_{0}\right]_{C}=\left[x_{0}\right]_{D},\left[x_{1}\right]_{C}=\left[x_{1}\right]_{D},\left[s y_{0} t\right]_{C}=\left[s y_{0} t\right]_{D} \text { and }\left[s^{\prime} y_{1} t^{\prime}\right]_{C}=\left[s^{\prime} y_{1} t^{\prime}\right]_{D} .
$$


We now deduce from the properties of $D$ that

$$
\left[x_{0}\right]_{C} \leq\left[s y_{0} t\right]_{C},\left[x_{1}\right]_{C} \leq\left[s^{\prime} y_{1} t^{\prime}\right]_{C} \text { and }[x]_{C}=\left[x_{0} x_{1}\right]_{C} \leq\left[s y_{0} t s^{\prime} y_{1} t^{\prime}\right]_{C} .
$$

Since $y_{0}$ and $y_{1}$ are consecutive, $t s^{\prime}$ is a loop, say around vertex $u$, so $\left[t s^{\prime}\right]_{C} \leq$ $1_{u}$ and $[x]_{C} \leq\left[s y_{0} y_{1} t^{\prime}\right]_{C}=\left[s y t^{\prime}\right]_{C}$. Similarly, since $x$ and $y$ are coterminal, $s$ and $t^{\prime}$ are loops, and we have $[x]_{C} \leq[y]_{C}$ as expected.

Now we assume that $x$ has no factorization $x=x_{0} x_{1}$ as above, but $x$ factors as $x=x_{0} a x_{1}$ with $\left[x_{0}\right]_{C},\left[x_{1}\right]_{C} \notin I \cup J$ and $a$ an arrow of $C$. Since $x \leq_{m}^{2} y, y$ can be factored either as $y=y_{0} a y_{1}$ or as $y=y_{0} y_{1}$ with $c_{r}\left(y_{0}\right) \subseteq c_{r}\left(x_{0}\right)$ and $c_{r}\left(y_{1}\right) \subseteq c_{r}\left(x_{1}\right)$ for each $r \leq m$. As above there exist paths $s, t, s^{\prime}, t^{\prime}$ in $C^{*}$ such that $[x]_{C} \leq\left[s y_{0} t a s^{\prime} y_{1} t^{\prime}\right]_{C}$ or $[x]_{C} \leq\left[s y_{0} t s^{\prime} y_{1} t^{\prime}\right]_{C}$. As above again, $s, t, s^{\prime}, t^{\prime}$ are loops in the first case, and $s, t s^{\prime}, t^{\prime}$ are loops in the second case. From which it follows that $[x]_{C} \leq\left[y_{0} a y_{1}\right]_{C}$ in the first case, and $[x]_{C} \leq\left[y_{0} y_{1}\right]_{C}$ in the second case: either way, we have $[x]_{C} \leq[y]_{C}$.

The last situation occurs if $x$ does not have a factorization of one of the above forms. Let $x_{0}$ be the longest prefix of $x$ such that $\left[x_{0}\right]_{C} \notin I \cup J: x_{0}$ may be empty, but it is different from $x$, so we have $x=x_{0} a x_{1}$ where $a$ is an arrow of $C$. By assumption, $\left[x_{0} a\right]_{C},\left[x_{1}\right]_{C} \in I \cup J$, so $[x]_{C}=\left[x_{0} a\right]_{C}\left[x_{1}\right]_{C} \in I$ and hence $[x]_{C} \leq[y]_{C}$. This concludes the proof.

Corollary 4.9 The variety $\mathbf{J}^{+}$is local.

Proof. Let $C$ be a category in $\ell \mathbf{J}^{+}$. Let $A$ be the set of all arrows of $C$. The free monoid $A^{*}$ can be viewed as the set of arrows of the free category over the graph with one object and with arrow set $A$. Let $m \geq 1$ and let $x, y \in A^{*}$. We say that $x \equiv_{m} y$ if $x \leq_{m} y$ and $y \leq_{m} x$. Then $\equiv_{m}$ is easily seen to be a finite index congruence on $A^{*}$. Let $J_{m}$ be the quotient monoid and let $\pi: A^{*} \rightarrow J_{m}$ be the natural morphism. Then letting $\pi(x) \leq \pi(y)$ whenever $x \leq_{m} y$ naturally equips $J_{m}$ with a structure of ordered monoid, such that $J_{m} \in \mathbf{J}^{+}$.

Now let $m \geq 1$ be such that $C \in \mathbf{V}_{m}$ : by Theorem 4.8, such an integer exists. Let $\delta: C \rightarrow J_{m}$ be the relation defined on each $C(u, v)$ by

$$
\delta(x)=\left\{\pi(y) \mid y \in C^{*}(u, v),[y]=x\right\} .
$$

Then $\delta$ is immediately verified to be a relational morphism. If $x, x^{\prime} \in$ $C^{*}(u, v)$ are such that there exist elements $z, z^{\prime}$, respectively in $\delta(x)$ and $\delta\left(x^{\prime}\right)$, such that $z \leq z^{\prime}$, then there exist paths $y, y^{\prime} \in C^{*}(u, v)$ such that $[y]=x,\left[y^{\prime}\right]=x^{\prime}$ and $y \leq_{m} y^{\prime}$ in $A^{*}$. Since $y$ and $y^{\prime}$ are coterminal paths in 
$C^{*}$, it follows that $y \leq_{m} y^{\prime}$ in $C^{*}$ and, by definition of $\mathbf{V}_{m}$, we have $x \leq x^{\prime}$. Thus $\delta$ is a division of ordered categories.

Thus $C \in \mathbf{g J}^{+}$, which completes the proof.

In [9], Pin and Weil introduced the variety (of semigroups) $\mathbf{B}_{1}^{+}$in the context of the study of the polynomial closure of certain classes of languages. As it turns out, $\mathbf{B}_{1}^{+}$is defined exactly to be $\mathbf{L J}^{+}$. Corollary 3.2 now yields the following.

Corollary 4.10 $\mathbf{B}_{1}^{+}=\mathbf{J}^{+} * \mathbf{D}$.

\section{Acknowledgements}

The authors would like to thank the anonymous referee for his many suggestions, which led to a significant improvement of the paper.

\section{References}

[1] J. Almeida and P. Weil, Profinite categories and semidirect products, J. Pure and Applied Algebra 123 (1998) 1-50.

[2] S. Eilenberg. Automata, languages and machines, vol. B, Academic Press, New York, 1976.

[3] P. R. Jones and S. Pustejovsky, A kernel for relational morphisms of categories, in Semigroups with Application, J.M. Howie, W.D. Munn and H.J. Weinert eds, World Scientific, Singapore, 1992, 152-161.

[4] R. Knast, A semigroup characterization of dot-depth one languages, RAIRO Inform. Théor. 17, (1983), 321-330.

[5] R. Knast, Some theorems on graph congruences, RAIRO Inform. Théor. 17, (1983), 331-342.

[6] S. Margolis, J.-E. Pin. Inverse semigroups and extensions of groups by semilattices, J. Algebra 110 (1987) 277-297.

[7] W.R. Nico. Wreath products and extensions, Houston J. Math 9 (1983) $71-99$.

[8] J.-E. Pin, A variety theorem without complementation, Izvestiya VUZ Matematika 39 (1995) 80-90. English version, Russian Mathem. (Iz. VUZ) 39 (1995) 74-83. 
[9] J.-E. Pin, P. Weil. Polynomial closure and unambiguous products, Theory of Computing Systems 30 (1997) 383-422.

[10] J.-E. Pin, P. Weil. Semidirect product of ordered semigroups, to appear.

[11] J.-E. Pin, P. Weil. The wreath product principle for ordered semigroups, to appear.

[12] J.-F. Rey. The block product of categories and Tilson's division, Fundamenta Informaticæ, 31, 1997, 379-400.

[13] J.-F. Rey. The kernel of a relational morphism of categories, Fundamenta Informaticæ, 31, 1997, 401-402.

[14] J.-F. Rey. The block product of $C$-varieties, comparing approaches, International Journal of Algebra and Computation, 9, 1999, 79-98.

[15] H. Straubing. Finite semigroup varieties of the form $\mathbf{V} * \mathbf{D}, J$. Pure Appl. Algebra 36 (1985) 205-215.

[16] M. Lurdes Teixeira. Estudo do produto semidirecto de pseudovariedades, Ph. D. Dissertation, Universidade do Porto, 1998.

[17] D. Thérien and A. Weiss. Graph congruences and wreath products, J. Pure Appl. Algebra 36 (1985) 205-215.

[18] D. Thérien. Catégories et langages de dot-depth un, Inform. Théor. Appl. 22 (1988) 437-445.

[19] B. Tilson. Complexity of semigroups and morphisms, in S. Eilenberg, Automata, Languages and Machines, vol. B, chap. XII, Academic Press, 1976.

[20] B. Tilson. Categories as algebras, J. Pure Appl. Algebra 48 (1987) 83-198. 